\title{
RNA Degradation
}

National Cancer Institute

\section{Source}

National Cancer Institute. RNA Degradation. NCI Thesaurus. Code C19144.

RNA degradation is a regulated cellular biochemical process that involves

depolymerization of RNA macromolecules, which affects the RNA content of cells and regulates gene expression. 